\title{
Mindfulness based treatment for smoking cessation: How it works and future directions
}

\author{
J. Kim Penberthy ${ }^{* 1}$, J. Morgan Penberthy ${ }^{2}$, Morgan Lynch ${ }^{1}$ and Dinesh Chhabra ${ }^{1}$ \\ ${ }^{1}$ Department of Psychiatry \& Neurobehavioral Sciences, University of Virginia School of Medicine, Charlottesville, VA, USA \\ ${ }^{2}$ Department of Psychology, Wake Forest University, Winston-Salem, NC, USA
}

In 2015, over 1.1 billion people smoked tobacco worldwide [1]. The World Health Organization (WHO) has estimated that tobacco use (smoking and smokeless) is responsible for the death of about six million people across the world each year [1]. This total includes about 600,000 people who are also estimated to die from the effects of second-hand smoke [1]. In the United States, smoking costs more than $\$ 193$ billion in health care costs and lost productivity per year [2]. Although over $70 \%$ of smokers want to quit, fewer than $5 \%$ achieve this goal annually [2]. Mainstay behavioral treatments for smoking have focused on teaching individuals to avoid cues, foster positive affective states, develop lifestyle changes that reduce stress, divert attention from cravings, substitute other activities for smoking, learn cognitive strategies that reduce negative mood, and develop social support mechanisms. These interventions and methods have shown modest success, with abstinence rates between only $20-30 \%$ holding steady over the past thirty years. This low rate of abstinence attainment and lack of improvement in outcomes is presumably due to the complex nature of the acquisition and maintenance of nicotine addiction, including associative learning mechanisms as well as positive and negative reinforcement.

The complex learning mechanisms behind the acquisition of nicotine dependence is related to the fact that over time cues that are judged to be positive or negative can induce affective states, which can then trigger a craving to use $[3,4]$. Though the centrality of craving remains controversial, evidence suggests that craving is strongly associated with using, which, mainly through the physiological properties of nicotine, results in the maintenance or improvement of positive or reduction of negative affective states [4]. This sets up reinforcement loops by reinforcing memories between affect and smoking [3]. Thus, recent attention has been focused on additional strategies to help people tolerate negative affect and cravings rather than only avoiding cues or substituting activities.

Recent research suggests that another psychological intervention called mindfulness training (MT) may decouple the association between craving and smoking, thus facilitating smoking cessation. Mindfulness means paying attention in the present moment, non-judgmentally, without commentary or decision-making. MT targets affective or craving states by teaching individuals to observe aversive body and mind states instead of responding to them with habitual reactions, thus allowing more adaptive, healthier responses [5]. MT has shown promise in reducing anxiety and depression and has recently been explored in the treatment of addictions. Davis et al. [6] conducted a pilot study to explore the effect of using Mindfulness Based Stress Reduction (MBSR) (with minor modifications) as a smoking intervention. MBSR was employed in its standard 8 weekly group session format.
Subjects attempted smoking cessation during week seven without pharmacotherapy. The researchers tested smoking abstinence after six weeks from the smoking quit day with a carbon monoxide breath test and 7-day smoking calendars. Prior to the intervention, the 18 subjects in the study had an average smoking history of 19.9 cigarettes per day for 26.4 years. At the 6 -week post-quit visit, $56 \%$ of subjects achieved biologically confirmed 7-day point-prevalent smoking abstinence. It was found that compliance with mindfulness meditation was positively associated with smoking abstinence and decreases in stress and affective distress. The results of this study suggest that mindfulness training shows promise as a therapy for smoking cessation.

A 2011 randomized controlled trial by Brewer et al. [7] provides further evidence for the effectiveness of mindfulness training for smoking cessation. In the study, 88 treatment-seeking, nicotinedependent adults smoking an average of 20 cigarettes per day were randomly assigned to MT or the American Lung Association's freedom from smoking (FFS) treatment. FFS is a behavior modification program and includes stress reduction and relapse prevention. The treatments had equal session visits and both were group formats. The primary outcome measures were biologically confirmed 7-day pointprevalence abstinence and number of cigarettes per day at the end of the treatment, and at follow-up 17-weeks after treatment. Treatment completion and follow-up completion rates were comparable between the two groups. Individuals who received MT reduced cigarette smoking more than those in the FFS treatment group, and they maintained these improvements at follow-up. Additionally, individuals who received $\mathrm{MT}$ achieved greater point prevalence abstinence at the end of treatment ( $36 \%$ vs. $15 \%$ ), and at the 17 -week follow-up ( $31 \%$ vs. $6 \%)$. Although both treatment groups were assigned home practice as part of their treatment, only those who received mindfulness training demonstrated a significant association between home practice and smoking outcomes, suggesting that there was a specific benefit to mindfulness practice and that positive treatment outcomes for those in this group are not merely a result of greater enthusiasm or interest in quitting.

These trials support the idea that mindfulness training may confer benefits greater than those associated with current standard treatments

Correspondence to: J. Kim Penberthy, Ph.D., ABPP, Chester F. Carlson Professor of Psychiatry \& Neurobehavioral Sciences, University of Virginia School of Medicine, Charlottesville, VA 22908 USA, Tel: 4349242241 ; Email: jkp2n@virginia.edu

Received: November 26, 2016; Accepted: December 24, 2016; Published: December 28, 2016 
for smoking cessation. The ability of MT to attenuate the relationship between craving and substance use has been observed in other studies as well. Elwafi et al. [8] demonstrated that people who practiced mindfulness more smoked less, regardless of their level of craving. Practicing mindfulness appears critical to MT treatment outcomes. These results suggest that MT may help individuals develop a tolerance to craving itself, thus over time acting to dismantle the addictive loop. To date, research in this area has been conducted in randomized clinical trials only in structured laboratory settings.

Important next steps are to examine the effectiveness of MT treatments for smoking cessation in naturalistic environments and to utilize methods of delivering treatment in real world settings that will facilitate compliance with mindfulness practice and thus improve treatment outcomes - for example, via a mobile device application. Additionally, trials comparing MT to alternative treatment approaches typically offered in outpatient clinics such as support groups, acupuncture, individual counseling, or nicotine replacement therapies are lacking. An estimated 11 million smokers in the U.S. own a smartphone, making applications (apps) that target smoking cessation a valuable, wide-spread, and easily accessible tool for individuals and researchers [9]. In 2012, 252 smoking-cessation applications existed for the iPhone, and 148 for the Android [9]. Abroms et al. [9] examined the content of 47 popular iPhone apps and 51 Android apps for smoking cessation that were distributed as of February 2012. Each app was analyzed and independently coded by two reviewers for its (i) approach to smoking cessation and (ii) adherence to the U.S. Public Health Service's Clinical Practice Guidelines for Treating Tobacco Use and Dependence. The apps analyzed were found to have low levels of adherence to key guidelines in the index. Few, if any, apps recommended or linked the user to proven treatments such as psychotherapy, counseling, and/or a Quitline. Additionally, for both iPhone and Android, apps with higher adherence index scores were rated more positively by users $(\mathrm{r}=0.29, \mathrm{p}<0.10 ; \mathrm{r}=0.32, \mathrm{p}<0.05$, respectively). This 2012 analysis is comparable to a 2009 analysis of smoking cessation apps, in which similar low adherence results were found, which indicates little if any progress has been made in improving popular smoking cessation apps [10]. We recommend that current apps be revised and future apps be developed around evidencebased practices for smoking cessation.

Despite the explosion of interest in the area of mobile device applications, research on both the design and potential uses of mindfulness-based mobile applications (MBMAs) is scarce. While a wide selection of MBMAs seem to be available to interested people, Plaza et al. [11] found an almost complete lack of evidence supporting the usefulness of those applications. They determined that the potential for mobile mindfulness applications remains largely unexplored. We propose that additional research is warranted on this potentially helpful intervention and that adaptations of mindfulness interventions, as well as additional psychological and behavioral interventions, must be made more broadly available to the public in order to facilitate a true positive impact on human health and well-being.

\section{References}

1. World Health Organization (2015) WHO global report on trends in prevalence of tobacco smoking 2015. Geneva: World Health Organization.

2. Ekpu VU, Brown AK (2015) The Economic Impact of Smoking and of Reducing Smoking Prevalence: Review of Evidence. Tob Use Insights 8: 1-35. [Crossref]

3. Baker TB, Piper ME, McCarthy DE, Majeskie MR, Fiore MC. 2004. Addiction motivation reformulated: An affective processing model of negative reinforcement. Psychol Review 111: 33-51. doi:10.1037/0033-295X.111.1.33.

4. Perkins K, Karelitz J, Conklin C, Sayette M, Gidgowd G (2010) Acute negative affect relief from smoking depends on the affect situation and measure but not on nicotine. Biol Psychiatry 67: 707-714. [Crossref]

5. Brewer JA, Elwafi HM, Davis JH (2013) Craving to quit: Psychological models and neurobiological mechanisms of mindfulness training as treatment for addictions. Psychol Addict Behav 27: 366-379. [Crossref]

6. Davis JM, Fleming MF, Bonus KA, Baker TB (2007) A pilot study on mindfulness based stress reduction for smokers. BMC Complement Altern Med 7: 2. [Crossref]

7. Brewer JA, Mallik S, Babuscio TA, Nich C, Johnson HE, et al. (2011) Mindfulness training for smoking cessation: Results from a randomized controlled trial. Drug Alcohol Depend 119: 72-80. [Crossref]

8. Elwafi HM, Witkiewitz K, Mallik S, Thornhill TA, Brewer JA (2013) Mindfulness training for smoking cessation: Moderation of the relationship between craving and cigarette use. Drug Alcohol Depend 130: 222-229. [Crossref]

9. Abroms LC, Lee Westmaas J, Bontemps-Jones J, Ramani R, Mellerson J (2013) A content analysis of popular smartphone apps for smoking cessation. Am J Prev Med 45 : 732-736. [Crossref]

10. Abroms LC, Padmanabhan N, Thaweethai L, Phillips T (2011) iPhone apps for smoking cessation: a content analysis. Am J Prev Med 40: 279-285. [Crossref]

11. Plaza I, Demarzo MM, Herrera-Mercadal P, Garcia-Campayo J (2013) Mindfulnessbased mobile applications: literature review and analysis of current features. JMIR Mhealth Uhealth 1: e24.

Copyright: (C2016 Kim Penberthy J. This is an open-access article distributed under the terms of the Creative Commons Attribution License, which permits unrestricted use, distribution, and reproduction in any medium, provided the original author and source are credited. 